\title{
Clinical features of spinal schwannomas in 65 patients with schwannomatosis compared with 831 with solitary schwannomas and 102 with neurofibromatosis Type \\ 2 : a retrospective study at a single institution
}

TO THE EDITOR: Thank you for publishing our article, "Clinical features of spinal schwannomas in 65 patients with schwannomatosis compared with 831 with solitary schwannomas and 102 with neurofibromatosis Type 2: a retrospective study at a single institution" (J Neurosurg Spine 24:145-154, January 2016).

After our article had been published online, we realized that the JNS Publishing Group's style of listing author affiliations does not conform with the requirements of our institution. We apologize for not noticing the difference in styles before publication, but we appreciate the flexibility shown by the JNS Publishing Group in allowing us to relist our authors and affiliations in the following manner:

Peng Li, MD, ${ }^{1} \mathrm{Fu}$ Zhao, MD, ${ }^{2}$ Jing Zhang, BE, ${ }^{2}$

Zhenmin Wang, MD, ${ }^{1}$ Xingchao Wang, MD ${ }^{1}$

Bo Wang, MD, ${ }^{1}$ Zhijun Yang, MD, ${ }^{1}$

Jun Yang, MD, ${ }^{1}$ Zhixian Gao, MD,${ }^{1}$ and

Pinan Liu, MD ${ }^{1}$

${ }^{1}$ Department of Neurosurgery, Beijing Tian Tan

Hospital, Capital Medical University, Beijing,

China; and ${ }^{2}$ Department of Neural Reconstruction,

Beijing Neurosurgery Institute, Capital Medical

University, Beijing, China

The authors' affiliation list has been corrected online as of May 27, 2016.

Pinan Liu, MD

Beijing Tian Tan Hospital, Capital Medical University, Beijing, China

INCLUDE WHEN CITING

Published online May 27, 2016; DOI: 10.3171/2016.5.SPINE141145a.

CAANS, 2016 